\title{
The Study of Solar Activity in Relation with High Frequency Variations of Solar Radio Flux
}

\author{
Antony Dhivya Tharshini S. , Shanthi G. \\ Department of Physics and Research Centre, Women's Christian College, Nagercoil, India
}

Email address:

tharshini.divya@gmail.com (Antony D. T. S.)

\section{To cite this article:}

Antony Dhivya Tharshini S., Shanthi G.. The Study of Solar Activity in Relation with High Frequency Variations of Solar Radio Flux. American Journal of Astronomy and Astrophysics. Vol. 3, No. 6, 2015, pp. 87-92. doi: 10.11648/j.ajaa.20150306.11

\begin{abstract}
In recent years, radio observations of solar emission at $10.7 \mathrm{~cm}$ wavelengths show a systematic increase compared to the sunspot number (SSN). The $10.7 \mathrm{~cm}$ solar radio flux (F10.7) is one of the most widely used indices of solar activity. The solar magnetic field flux measured for the entire solar cycle is compared with the solar index of the effective cycles for the years 1986-2015. The solar activity indices include numerous quantities. Of these indices the two most used are the relative sunspot number, covering more than 300 years and the $10.7 \mathrm{~cm}$ solar radio flux (F10.7), which has been measured regularly since 1947 . The data published in the official website of National Oceanic and Atmospheric Administration (NOAA) has been used in the analysis. This study examines the relative sunspot number and $10.7 \mathrm{~cm}$ solar radio flux indices individually and also the relation between them, and also what it implies on the solar behaviour for the solar cycle 22, 23 \& 24.
\end{abstract}

Keywords: SSN, Solar Radio Flux, Solar Activity, Indices, Data Analysis

\section{Introduction}

The $10.7 \mathrm{~cm}$ solar flux is widely used for upper atmosphere studies as an index of the solar extreme ultraviolet radiation (EUV), whose variations are responsible for major changes in properties above $200 \mathrm{~km}$. F10.7 is a general indicator of solar magnetic activity, solar ultraviolet and $\mathrm{X}$ - ray emissions, and even solar irradiance. F10.7is used for a wide range of applications including astronomy, climate modelling, geophysics, meteorology, communications, and satellite systems and so on (Albert Anderson 1964; Mathieu Barthelemy et al 2014). The microwave flux at $10.7 \mathrm{~cm}$ has been routinely used as a solar proxy; shows that the radio fluxes at other wavelengths provide valuable complementary information that enhances their value for upper atmospheric modelling. (Thierry Dudok de Wit et al 2014) As a proxy for solar extreme ultraviolet (EUV) radiation, F10.7 is always an input parameter for calculating the atmospheric density for orbit determination in aerospace and predicting the influence of the ionosphere on communication. (Cong Huang et al 2009; John Xanthakis \& Constantine Poulakos, 1985; Matthieu Kretzschmar et al 2012)

The space weather discipline involves different physical scenarios, which are characterised by very different physical conditions, ranging from the sun to the terrestrial magnetosphere and ionosphere (Elena Saiz et al 2013). The sun emits radio energy with slowly varying intensity. The radio flux, which originates from atmospheric layers are high in the sun's chromospheres and low in its corona, changes gradually from day to day in response to the number of spot groups on the disk. Solar flux from the entire solar disk at a frequency of $2800 \mathrm{MHz}$ is a density per unit frequency at a wavelength of $10.7 \mathrm{~cm}$, near the peak of the observed solar radio emission. It represents a measure of diffuse, non-radiative heating of the coronal plasma trapped by magnetic fields over active regions, and is an excellent indicator of overall solar activity levels (Saha et al 2011). The $10.7 \mathrm{~cm}(2.8 \mathrm{GHz})$ electromagnetic band is of much importance because it can be used as a good substitute for ground-based observations such as those in other radio bands and space-borne observations such as in the UV band (Chatterjee 2001). The $2.8 \mathrm{GHz}(10.7 \mathrm{~cm})$ radio emission, which has been used as an indicator of solar activity for various activity for various kinds of solar terrestrial effects. This radio emission, as well as the total emission in other parts of the electromagnetic spectrum, has been found to have a 27-day cyclic variation, known as the slowly varying or sunspot dependent component of the radio emission.(Chatterjee \& Das 1995)

A sunspot is a phenomenon observed in the solar photosphere. The chromospheres are a layer above the 
photosphere. The corona is the outermost atmosphere of the sun. The electromagnetic radiation of the sun at a frequency of $2800 \mathrm{MHz}$ having wavelength $10.7 \mathrm{~cm}$ is an important parameter for measuring the level of solar activity. The intensity of solar F10.7is expressed by solar radiation flux at a wavelength of $10.7 \mathrm{~cm}$. Observations of $10.7 \mathrm{~cm}$ began in 1947 and are available for more than 60 years. The value of solar activity is low and high when solar activity is high, with the correlation between the solar F10.7and relative sunspot number exceeds 0.98. ( David Hathaway et al 2003). The solar F10.7reflects the activity of the corona, while the relative sunspot number mirrors the activity of the photosphere. It has been questioned whether the levels of solar atmospheric activity in two different layers agree with one another considering that relative sunspot numbers and F10.7are two different series, one has to analyze the phase relationship between the two using different methodologies.(ZHANG XueFeng et al 2012; Ivan Kutiev et al 2013)

Magnetic activity of the sun is called the complex of electromagnetic and hydrodynamic processes in the solar atmosphere. The analysis of active regions is required to study the magnetic field of the sun and the physics of magnetic activity. This task is of fundamental importance for astrophysics of the sun and the stars. Its applied meaning is connected with the influence of solar active processes on the Earth's magnetic field.(Bruevich et al 2014). The consensus reconstruction shows reasonable agreement among the various reconstructions of solar wind magnetic field the past 170 years (Leif Svalgaard 2013). During a roughly 11 year period, the number of sunspots seen on the solar disk shows a cyclic change. The current sunspot cycle (cycle 24) has been strangely slow to develop, but even more strange is that infrared measurements of the central dark sunspot umbral regions have shown a decrease in the maximum magnetic field strength.(Livingston et al 2012; Varotsos et al 1992).The purpose of this study is to present the variation of sunspot number with the variable frequency of solar radio flux for minimum and maximum solar cycle.

\section{Data Analysis}

The data published in the Solar Geophysical Data Bulletins in the website of NOAA (fttp://fttp.ngdc.noaa.gov/STP/SOLAR_DATA/....html) available in public domain. The US air force operates four Radio Solar Telescope Network (RSTN) observatory monitors. Solar radio emissions on 8 discrete fixed frequencies such as $245 \mathrm{MHz}, 410 \mathrm{MHZ}, 610 \mathrm{MHz}, 1415 \mathrm{MHZ}, 2695 \mathrm{MHz}, 4995$ MHZ, $8800 \mathrm{MHz}$ and $15400 \mathrm{MHz}$. In this study we adopt the daily values of above mentioned frequencies from Learmonth solar Radio spectrograph for the $22^{\text {nd }}$ solar cycle from 1986 to 1996.The daily data of $2800 \mathrm{MHz}$ radio emission, as well as that of the relative sunspot number for the same period, have been obtained from the same publications. The time series of the monthly mean values of solar indices were used in the analyses. The sun spot numbers were received from the website (ftp://ftp.ngdc.noaa.gov/STP/space-weather/solar-data/solar-in dices/sunspotnumber/).

The data of the $10.7 \mathrm{~cm}$ solar radio flux were obtained from the website (ftp://ftp.ips.gov.au/wdcdata/solradio/data/learmonth/). The appearance of a solar cycle was a random event. The solar radio flux is an excellent indicator of overall solar active. The solar radio flux can be measured relatively easily and is used as an index of solar activity for many purposes.

\section{Results and Discussion}

The analysis creating visible sunspots are varied. And indeed, while there has been a long stable relationship between the $10.7 \mathrm{~cm}$ flux and the sunspot number, allowing one to calculate or map one from the other, that relationship has steadily deteriorated in the past decade to the point where the sunspot number for a given flux has gone down by about a third (Fig 1). The long term variation of the solar flux for $22^{\text {nd }}$ solar cycle is plotted for the period 1986-1996 along with the corresponding frequencies $(245 \mathrm{MHz}-15400 \mathrm{MHz})$ of solar radio flux. It is clear that the well-understood physics of causes of the diurnal variation of the geomagnetic field provides a reliable way of assessing the past variation of solar flux, and hence the magnetic activity responsible for it, a variation for which the sunspot number is a proxy. Careful application of this method fully supports the two adjustments of the sunspot series described above based on the diurnal variation as observed since $1780 \mathrm{~s}$. The variation of $22 \mathrm{nd}$ solar cycle which is depicted as a plot between solar flux and year for the entire cycle with all the eight frequencieshas been analysed and plotted (Fig 2).

The data are grouped into 12 month bins with all the available hourly data within the respective month of the year. In addition to the absolute level of frequencies, the information that we seek here is the origin of the solar variability. The lack of purity makes however the attribution of this variability very delicate. As the variability of the solar activity is wavelength dependent, for the entire solar cycle. The multiple peaks in the plot show the significant differences. However it may be noted that the maximum is noticed during the initial stage of the solar cycle for $245 \mathrm{MHz}$ with the wavelength $122 \mathrm{~cm}$.

A relatively larger deviation is observed in amplitude whose reconstruction is therefore more easily compromised by the impact of solar radio flux. Interestingly, the solar flux shows significant multiple peaks between this time periods. From the figure it can be seen that the solar flux for the frequency 610 $\mathrm{MHz}$, there is a gradual rise during 1987- 1990. In particular, for the F10.7 cm flux the variability of the amplitude can be more easily ascribed to that of specific solar activity. Finally, the approaches we have presented have offers an interesting tool for evaluating the performance with different frequencies. It is evident that the amplitude distribution shows some peaks corresponding to $10.7 \mathrm{~cm}$ solar radio flux maximum during the year 1989-1990 close to the solar activity maximum years. Comparisons between these indices afford a subtle means to detect changes in the solar activity cycle. 

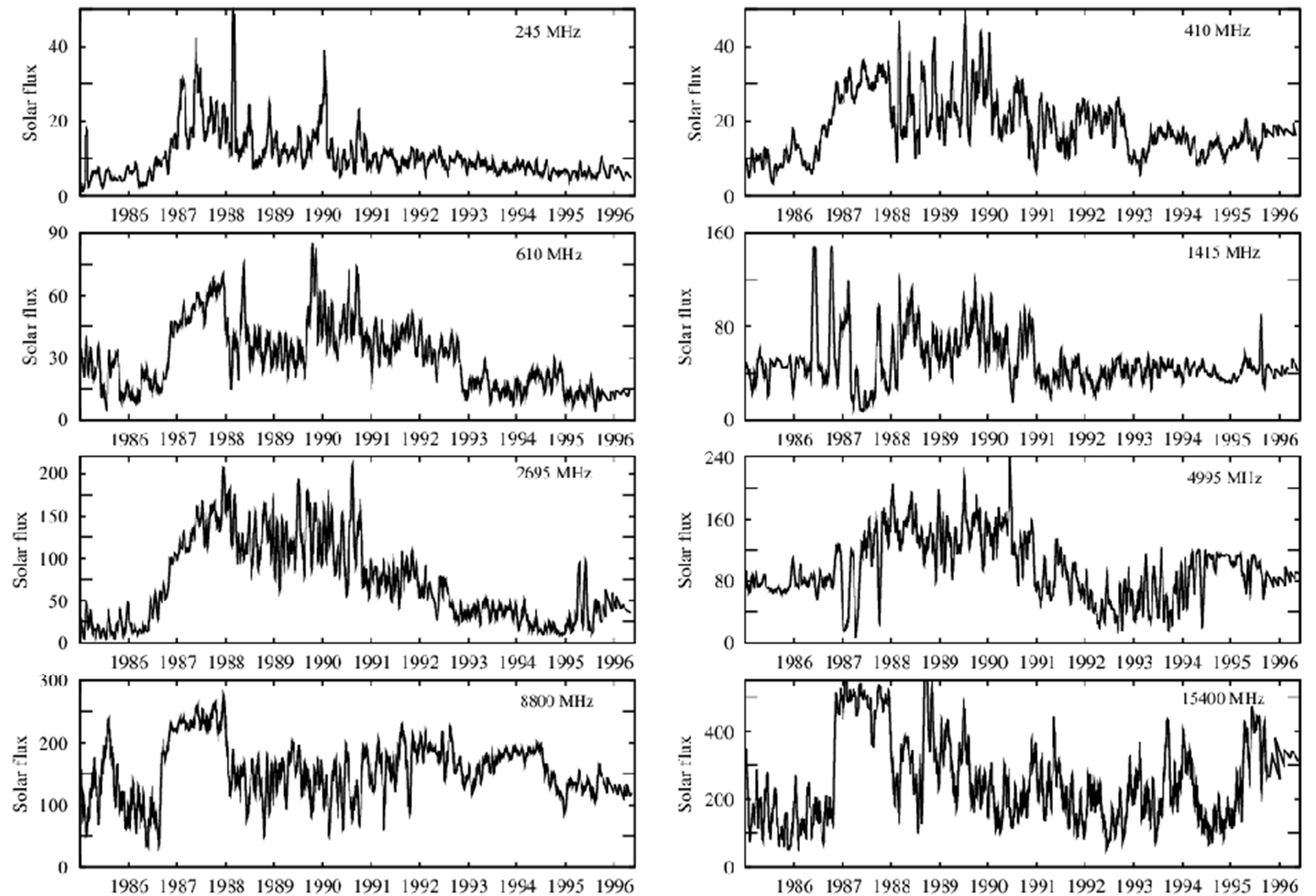

Year

Figure 1. Time series utilized for this investigation from September 1986 to May 1996 at daily resolution. The difference between the corresponding frequencies contributes to the shorter wavelength for the frequency $245 \mathrm{MHz}$ to the longer wavelength for the frequency $15400 \mathrm{MHz}$. The maximum values of the radio flux is $575 \mathrm{sfu}\left(1 \mathrm{sfu}=10^{-22} \mathrm{Wm}^{-2} \mathrm{~Hz}^{-1}\right)$ for the frequency $15400 \mathrm{MHz}$.

The 11 year modulations are also properly reconstructed, except for the few peaks whose variability is not fully captured by the observatory monitors. Globally, the solar radio flux up to $15400 \mathrm{MHz}$ is quite satisfactorily reconstructed and is largely within the instrumental precision, except for some wavelengths. Further investigations are needed to confirm such results with future data sets and in particular with independent instruments, such as Extreme Ultraviolet Variability Experiment (EVE) on board with Solar Dynamics Observatory (SDO) and Radio Solar Telescope Network (RSTN). Such an instrument with broadband measurements is particularly appropriate for monitoring day to day variations in the solar radio flux.

The phenomena of the relative importance of different frequencies are not the same for all observed frequency at the solar radio flux. Whereas figure (3) depicts their variations in the hourly mean solar radio flux from 1986 to the near end of 1996 for the 23 and 24 solar cycles. Solar radio fluxes around $3 \mathrm{GHz}$ show similar behaviour, confirming the existence of a close relation between $\mathrm{R}$ and the radio flux at $2.8 \mathrm{GHz}$.

The mean daily frequency of the solar radio flux is comparatively high in order to validate these time series. The SDO/RSTN data over the period after 1988 with the solar radio flux from $20 \mathrm{sfu}$ to $210 \mathrm{sfu}$. A daily minimum value of F10.7 $\mathrm{cm}$ has been retrieved by hand after the examination of the daily curve; this has been done for the purpose of radio flux studies, but we use it here to validate the daily mean time series. Indeed the agreement between the amplitude is excellent and shows the reliability of our daily value. The maximum values of those radio fluxes are $49 \mathrm{sfu}, 50 \mathrm{sfu}, 8$ sfu, $145 \mathrm{sfu}, 210 \mathrm{sfu}, 250 \mathrm{sfu}, 275 \mathrm{sfu}, 575 \mathrm{sfu}\left(1 \mathrm{sfu}=10^{-22} \mathrm{~W} \mathrm{~m}^{-2}\right.$ $\mathrm{Hz}^{-1}$ ) respectively.

The solar F10.7 emission used in RSTN as a proxy for UV/EUV emissions, which heat the upper atmosphere of the Earth. However due to the strong variability of the $10.7 \mathrm{~cm}$ flux, which is really important for space weather purposes, they also need good accuracies and stabilities. This study also underlines the need for long-term measurements with high resolution line profiles and large wavelength coverage.

The above graph illustrates the behaviour of the solar radio flux at $10.7 \mathrm{~cm}$ from the year 1986 to 1996 . The $10.7 \mathrm{~cm}$ solar radio flux start increasing from 1986 (solar activity minimum) 
and reaches to its first maximum (210) during 1989 and then reaches to its minimum on 1990 , then increases sharply and reaches to its second maximum during 1991 . The $10.7 \mathrm{~cm}$ solar radio flux gradually decreases from the year 1991 to 1995.
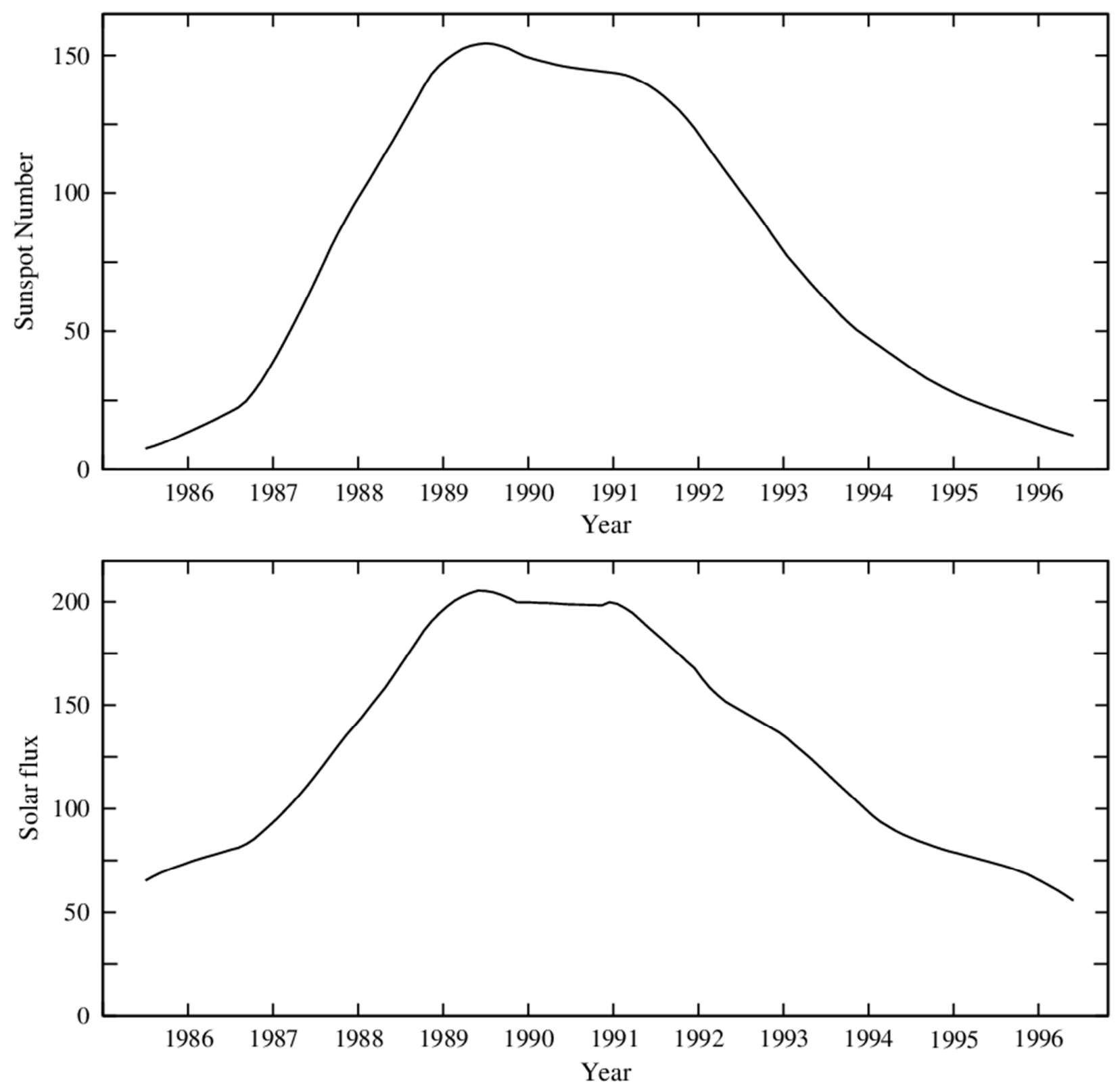

Figure 2. Mean amplitude of the 11 year solar cycle (solar cycle 22) modulation observed for the year (1986-1996). The upper plot is for sunspot number and lower plot is for solar flux and their reconstruction with the $10.7 \mathrm{~cm}$ flux only. The time series is identical which have been normalised to their maximum value in order to ease visualisation.

Table 1. Sunspot number vs $F_{10.7}$ : Observational data 1986-1996. Statistics of the model comparisons with the sunspot number and 10.7 $\mathrm{cm}$ radio flux. Each pair of values stands for the result obtained with the solar cycle 22.

\begin{tabular}{|c|c|c|c|c|c|}
\hline Wavelength (m) & Frequency (MHz) & Origin of observations & Beginning of measurements & $\begin{array}{l}\text { Number of peaks } \\
\text { since beginning }\end{array}$ & Average level (sfu) \\
\hline 1.220 & 245 & & \multirow{8}{*}{ September 1986} & 4 & 49 \\
\hline 0.731 & 410 & & & 6 & 50 \\
\hline 0.491 & 610 & Learmonth & & 5 & 80 \\
\hline 0.212 & 1415 & Solar Radio & & 8 & 145 \\
\hline 0.113 & 2695 & Spectrograph & & 6 & 210 \\
\hline 0.060 & 4995 & & & 5 & 250 \\
\hline 0.034 & 8800 & & & 3 & 275 \\
\hline 0.019 & 15400 & & & 4 & 575 \\
\hline
\end{tabular}



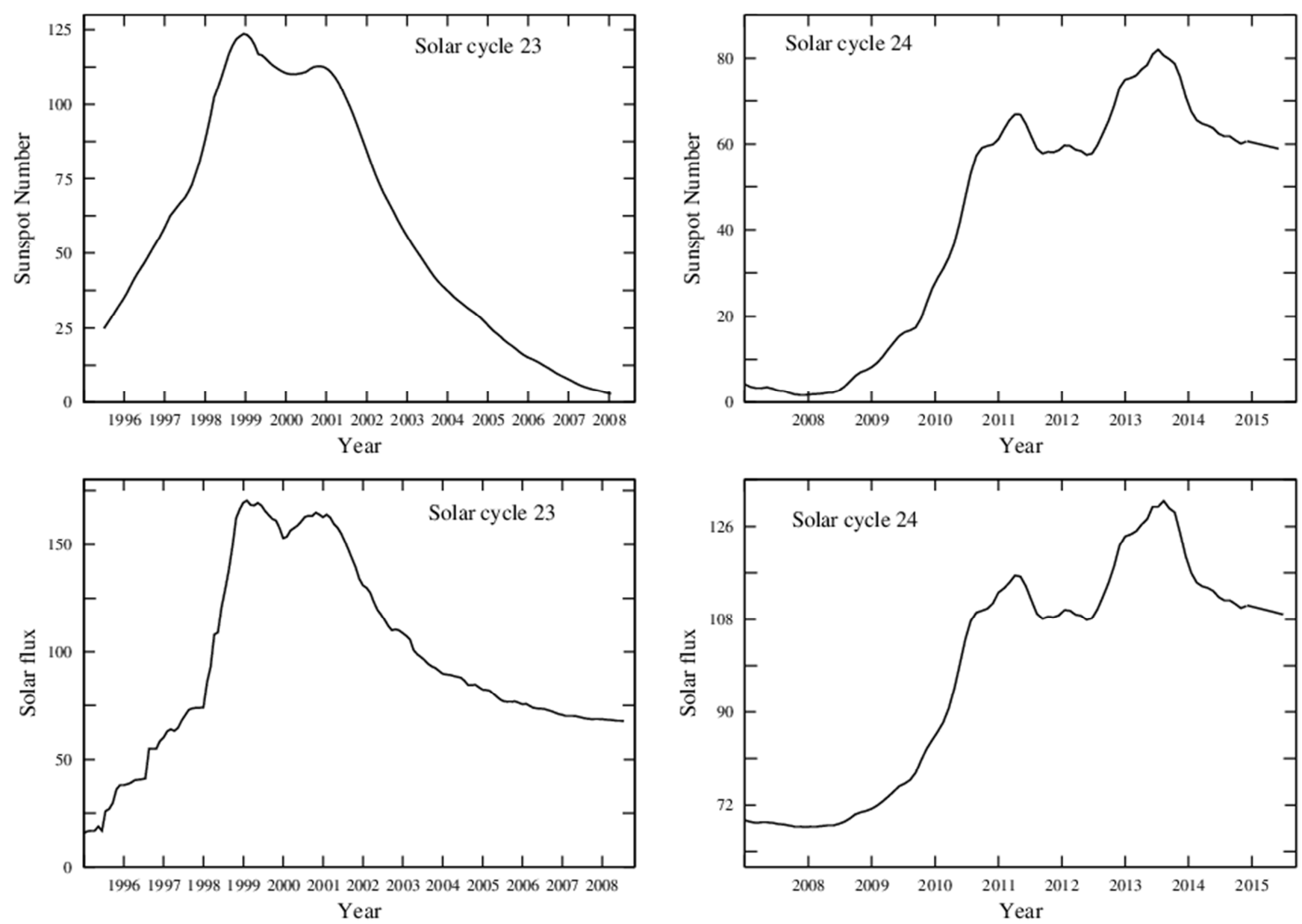

Figure 3. Correlation coefficient between the sunspot number (SSN) and the solar flux at various wavelengths for the solar cycle 23-24. Best fit refers to the correlation coefficient obtained with the linear combination of wavelengths, respectively sources, that best matches the SSN.

The Learmonth Solar Radio spectrograph monitors the sun in multiple frequencies. Here we consider observations made at $0.019 \mathrm{~m}, 0.034 \mathrm{~m}$ and $1.22 \mathrm{~m}$ only. Shorter wavelengths are observed as well, but we ignore them because of their escalating sensitivity to weather conditions. Observations began on September 1986, see Table 1. Observations at different frequency were interrupted as the antennas were moved from their location. All instruments operate continuously during daytime. Once per month, flux corrected daily averages are also automatically published on a daily basis. These fluxes are not normalised to a constant Sun-Earth distance. The monthly mean indices for $10.7 \mathrm{~cm}$ radio flux variation with the relative sunspot number are plotted in figure (3). First half demonstrates the observed data of sunspot number and the second half shows the F10.7 for the solar cycle $23-24$ which ensures the dramatic flip in the magnetic field.

\section{Conclusion}

The foremost conclusion of this study is the superior performance of sunspot number (SSN) over the radio flux 10.7 $\mathrm{cm}$. When it comes to modelling the response of the upper atmosphere on time scales of days beyond. We have built a homogenous and continuous data set of daily solar radio emission at eight frequencies. Such synoptic observations provide considerable added value over the sole F10.7 index because the relative contribution of various emission processes is not same in all aspects. Reconstructions of various solar proxies, and of SSN observations with these eight radio fluxes shows that the best trade off between the model complexity and correlation is obtained when a combination of radio frequencies used. In this paper, we have presented the data in order to build daily time series over the mission lifetime since 1986 to show the stability over years. Our approach is based on the observational evidence that most of the solar indices variability can be reconstructed from the measurement of different indices.

The results described in the paper are products on the data driven analysis. The availability, quality and coherence of the data are primary factors determining the value of obtained results. The simple interpretation of the periodic variations makes the solar cycle 22 exceptionally with dual peaks during the year 1989 and 1991. The possible, recent reassessment of past solar activity should provide a better theoretical review for solar cycle 23 and solar cycle 24 . If the discrepancy between the sunspot number and other indices continues, the leading sunspots shows cyclic behaviour of different solar radio fluxes and sunspot number variation during solar activity cycle. The day by day comparison of the $10.7 \mathrm{~cm}$ 
radio flux values and the relative sunspot numbers emphasizes that the $10.7 \mathrm{~cm}$ radio flux values are responding to the magnetic field which intensify the sunspots.

\section{References}

[1] Albert. D. Anderson.,(1964), On the Inexactness of the $10.7 \mathrm{~cm}$ flux from the sun as an Index of the total extreme ultraviolet radiation, Journal of the Atmospheric Sciences. 21, 1-14.

[2] Bruevich. E.A., Bruevich. V.V and Yakunina. G.V.,(2014), Changed relation between solar $10.7 \mathrm{~cm}$ radio flux and some activity indices which describe the radiation at different altitudes of atmosphere during cycles 21-23, Journal of Astrophysics and Astronomy.35,1-15.

[3] Chatterjee. T.N.,(2001), On the application of information theory to the optimum state-space reconstruction of the short-term solar radio flux $(10.7 \mathrm{~cm})$, and its prediction via a neural network, RAS. 323,101-108.

[4] Chatterjee. T.N and Das. T.K.,(1995), Relation between solar UV flux and $10.7 \mathrm{~cm}$ radio emission, RAS. $274,858-860$.

[5] Cong Huang., Dan-Dan Liu and Jing- Song Wang.,(2009), Forecast daily indices of solar activity, F10.7, using support vector regression method, Research in Astron. Astrophys. 9, 694-702.

[6] David. H. Hathaway., Dibyendu Nandy., Robert. M. Wilson and Edwin. J. Reichmann.,(2003), Evidence that a deep meridional flow sets the sunspot cycle period, The Astrophysical Journal. 589,665-670.

[7] Elena Saiz., Yolanda Cerrato., Consuelo Cid., Venera Dobrica., Pavel Hejda., Petko Nenovski., Peter staining., Josef Bochnicek., Dimitar Danov., Crisan Demetrescu., Walter D. Gonzalez., Georgeta Maris., Dimitar Teodosiev and Fridich Valach., (2013), Geomagnetic response to solar and interplanetary disturbances, Journal of Space Weather Space Climate. DOI: $10.1051 / \mathrm{swsc} / 2013048$.

[8] Ivan Kutiev., Ioanna Tsagouri., Loredana Perrone., Dora Pancheva., Plamen Mukhtarov., Andrei Mikhailov., Jan Lastovicka., Norbert Jakowski., Dalia Buresova., Estefania
Blanch., Borislav Andonov., David Altadill., Sergio Magdaleno., Mario Parisi and Joan Miquel Torta., (2013), Solar activity impact on the Earth's upper atmosphere, Journal of Space Weather Space Climate. DOI: 10.1051/swsc/2013028.

[9] John Xanthakis and Constantine Poulakos.,(1985), Long and short term variation of the $10.7 \mathrm{~cm}$ solar flux. The photospheric granules and the Zurich Numbers, Journal of Astrophysics and Space Science. 111,179-188.

[10] Leif Svalgaard., (2013), Solar activity - past, present, future, Journal of Space Weather Space Climate. DOI: $10.1051 / \mathrm{swsc} / 2013046$.

[11] Livingston. W., Penn. M.J and Svalgaard. L.,(2012) Decreasing sunspot magnetic fields explain unique $10.7 \mathrm{~cm}$ radio flux, The Astrophysical Journal Letters.757,1-4.

[12] Mathieu Barthelemy and Gael Cessateur., (2014), Sensitivity of upper atmospheric emissions calculations to solar/stellar UV flux, Journal of Space Weather Space Climate. DOI:10.1051/swsc/2014033.

[13] Matthieu Kretzschmar., Ingolf E. Dammasch., Marie Dominique., Joe Zender., Gael Cessateur and Elke D Huys., (2012), Extreme ultraviolet solar irradiance during the rising phase of solar cycle 24 observed by PROBA2/LYRA, Journal of Space Weather Space Climate. DOI: 10.1051/swsc/2012014.

[14] Saha. U, M.Sc., Midya. S.K, Ph.D and Das. G.K, M.Sc.,(2011), The effect of the variable component of $10.7 \mathrm{~cm}$ solar flux on the thunderstorm frequency over Kolkata and its relation with ozone depletion mechanism, The Pacific Journal of Science and Technology.12, 591- 597.

[15] Thierry Dudok de Wit., Sean Bruinsma and Kiyoto Shibasaki., (2014), Synoptic radio observations as proxies for upper atmosphere modeling, Journal of Space Weather Space Climate. DOI: $10.1051 / \mathrm{swsc} / 2014003$.

[16] Varotsos. C.A, Dris. N.A, Asimakopoulos. D.N and Cartalis. C.,(1992), On the relationship between the $10.7 \mathrm{~cm}$ solar flux, surface pressure and air temperature over Greece, Journal of Theor.Appl.Climatol.46,27-32.

[17] Zhang Xue Feng., LE Gui Ming and Zhang Yan Xia.,(2012), Phase relationship between the relative sunspot number and solar 10.7cm flux, Chin Sci Bull. 57, 2078-2082. 